\title{
Self-Reported Physical Activity and Myocardial Flow Reserve in Postmenopausal Women at Risk for Cardiovascular Disease
}

\author{
CLAIRE S. DUVERNOY, M.D., ${ }^{1}$ JULIE W. MARTIN, M.D., ${ }^{1}$ KERRI BRIESMIESTER, B.S., ${ }^{1}$ \\ OTTO MUZIK, Ph.D., ${ }^{2}$ and LORI MOSCA, M.D., Ph.D., M.P.H. ${ }^{3}$
}

\begin{abstract}
Background: Regular exercise protects against coronary heart disease (CHD) events and improves vascular reactivity. Exercise effects on myocardial flow reserve (MFR) are not well studied.

Methods: We performed dynamic N-13 ammonia positron emission tomography (PET) in 16 postmenopausal women ( $60 \pm 6$ years) to measure myocardial blood flow (MBF) and MFR. We also obtained information from each woman on her self-reported physical activity.

Results: Of the 16 patients, 6 reported moderate regular physical activity, and 10 did not. Women who reported regular, at least moderate physical activity had a higher percentage increase in adenosine $\mathrm{MBF}$ from rest compared with women who did not exercise $(268 \%$ vs. $129 \%, p=0.04)$ and had a significantly higher mean maximal MFR (3.68 vs. 2.29, $p=0.04)$.

Conclusions: These findings provide further mechanistic support for the beneficial cardiovascular effects of exercise.
\end{abstract}

\section{INTRODUCTION}

$\mathbf{R}$ EGULAR EXERCISE HAS BEEN ASSOCIATED with improved cardiovascular outcomes and has been shown to improve vascular reactivity. ${ }^{1,2} \mathrm{We}$ hypothesized that the beneficial effects of regular exercise would be associated with improved vascular reactivity, as measured by maximal myocardial blood flow (MBF) and myocardial flow reserve (MFR), when compared with individuals who did not engage in regular exercise. Because invasive coronary flow measurements are difficult to justify in healthy subjects, we sought to use a noninvasive technique to measure microcirculatory vascular reactivity and MBF.

\section{MATERIALS AND METHODS}

Subjects included postmenopausal (without menses for $\geq 12$ months) women with at least one coronary heart disease (CHD) risk factor $(n=16)$.

${ }^{1}$ Division of Cardiology University of Michigan Ann Arbor, Michigan, and Cardiology Section, VA Ann Arbor Healthcare System Ann Arbor, Michigan.

${ }^{2}$ Nuclear Medicine, Wayne State University PET Center, Detroit, Michigan.

3Preventive Cardiology, New York-Presbyterian/Columbia University Medical Center, New York, New York.

This project was sponsored by a grant from the Society for Women's Health Research and Pfizer, Inc., and by the Veterans Affairs Ann Arbor Health System.

Presented as a poster at the American Heart Association Scientific Sessions 2002 on November 17, 2002, under the title, Physical Activity Improves Myocardial Flow Reserve in Healthy Postmenopausal Women at Risk for Coronary Heart Disease. 
Study subjects were recruited from the VA Ann Arbor Women's Health Clinic or through community newspaper advertisement. Subjects were excluded if they had evidence of CHD, recent $(<3$ months) initiation of lipid-lowering therapy or use of hormone replacement therapy (HRT), inability to discontinue vasoactive medicines for 24 hours prior to positron emission tomography (PET), and inability to give informed consent. All subjects underwent dobutamine stress echocardiography to exclude CHD. Each woman recruited for the study provided information about her health and activities, including marital status, education level, employment status, alcohol intake, weight, weight changes, dietary habits, menstrual history, and physical activity. Physical activity was assessed using a modified Paffenbarger Physical Activity Questionnaire, which has been used by members of our research group in the past. ${ }^{3,4}$ Women were required to note the physical demands of their usual daily activity (not at all, partly, moderately, or very demanding), answer yes or no to the question, "At least once a week, do you engage in any regular physical activity (brisk walking, jogging, bicycling, etc.) long enough to work up a sweat?" and give the number of times per week and number of minutes per time. The women were further asked to note how many flights of stairs climbed per day, how many city blocks walked each day, how many years they had engaged in regular exercise, and during which seasons they performed regular exercise. For the purposes of the present analysis, women were divided into two categories based on their answers to the questions: those who reported exercise of at least 30 minutes per day at least twice per week continuously throughout the year, and those who reported less than that amount of physical activity. All subjects underwent dynamic PET. MBF was measured using a three-compartment model at rest, during cold pressor testing $(\mathrm{CPT})$, and after adenosine infusion in order to determine baseline, sympathetic stimulus-dependent, and maximal flows, respectively. The study protocol was approved by the institutional review boards of the University of Michigan and the Ann Arbor Veterans Affairs Medical Center. The study was also approved by the Radiation Safety Committee. Each subject gave informed consent. PET studies were acquired, processed, and analyzed as has been previously reported. ${ }^{5} \mathrm{MBF}$ was measured at rest, during CPT (in order to measure blood flow during sympathetic stimulation), and after intra- venous (i.v.) adenosine infusion in order to measure maximal flow. MFR was defined as the ratio between MBF in response to adenosine and $\mathrm{MBF}$ at rest.

Summary statistics of groups are presented as mean \pm standard deviation (SD). A two-tailed Student's $t$ test was used to compare values between groups. All variables were plotted to determine whether or not they were normally distributed. For variables that were not normally distributed (triglycerides and high-density lipoprotein [HDL]cholesterol), a two-sample Wilcoxon rank sum test was used to compare the values between groups. Pearson correlation coefficients were calculated for continuous variables. A chi-square or Fisher's exact test was used to compare discrete variables, as appropriate; $p$ values $<0.05$ were considered significant. All analyses were performed with the SPSS for Windows, version 11.0 statistics package, (SPSS, Chicago, IL).

\section{RESULTS}

Sixteen women were enrolled and underwent N-13 ammonia PET studies at rest, with CPT, and with adenosine. Health questionnaire data were available from all. Lipid levels were available in 15 women.

The mean age of the 16 women was $60 \pm 6$ years. Other CHD risk factors and medication use are summarized in Table 1. In the study group as a whole, 5 of the 16 women had two CHD risk factors, 5 had three risk factors, 5 had four risk factors, and 1 had six risk factors. Breakdown by physical activity group is given in Table 1 .

Myocardial blood flow rose significantly after adenosine infusion and did not change significantly from rest during the CPT. Blood flow and cardiac workload values for each subject are shown in Table 2. Mean rate pressure product (RPP), MBF and MFR are shown in Table 3. With the exception of low-density lipoprotein (LDL)cholesterol, which was paradoxically higher in the active group (possibly due to somewhat higher triglycerides in the inactive group, which would reduce calculated LDL values), we found no correlation between traditional risk factors in our population (total cholesterol, LDL, HDL, triglycerides, smoking, hypertension, family history of premature $\mathrm{CHD}$, and diabetes) and MBF measurements. Weight and dieting history were found to correlate significantly with $\mathrm{MBF}$ during $\mathrm{CPT}$, as we have previously reported. ${ }^{6}$ We fur- 
Table 1. Patient Characteristics ${ }^{\mathrm{a}}$

\begin{tabular}{|c|c|c|c|}
\hline & $\begin{array}{c}\text { Active group } \\
n=6\end{array}$ & $\begin{array}{c}\text { Inactive group } \\
n=10\end{array}$ & pvalue \\
\hline Age, years & $58 \pm 5$ & $62 \pm 7$ & 0.12 \\
\hline Total cholesterol (mg/dL) & $249 \pm 30$ & $228 \pm 51$ & 0.41 \\
\hline Triglycerides (mg/dL) & $115 \pm 24$ & $211 \pm 154$ & 0.22 \\
\hline Low-density lipoprotein (LDL)-cholesterol (mg/dL) & $171 \pm 24$ & $133 \pm 26$ & 0.02 \\
\hline High-density lipoprotein (HDL)-cholesterol (mg/dL) & $55 \pm 6$ & $53 \pm 23$ & 0.18 \\
\hline Body mass index $\left(\mathrm{kg} / \mathrm{m}^{2}\right)$ & $28.0 \pm 4$ & $31 \pm 4$ & 0.08 \\
\hline $\begin{array}{l}\text { Hypercholesterolemia }(\mathrm{TC}>200 \mathrm{mg} / \mathrm{dL}, \\
\text { LCL }>130 \mathrm{mg} / \mathrm{dL} \text {, or current statin use) }\end{array}$ & $6(100 \%)$ & $9(90 \%)$ & 0.46 \\
\hline Hypertension & $2(34 \%)$ & $3(30 \%)$ & 0.90 \\
\hline Diabetes & $0(0 \%)$ & $2(20 \%)$ & 0.27 \\
\hline Family history of premature coronary disease & $3(50 \%)$ & $6(60 \%)$ & 0.72 \\
\hline Smoking & $4(67 \%)$ & $5(50 \%)$ & 0.55 \\
\hline Obese $(\mathrm{BMI} \geq 30)$ & $2(34 \%)$ & $6(60 \%)$ & 0.17 \\
\hline Angiotensin-converting enzyme inhibitor use & $1(17 \%)$ & $1(10 \%)$ & 0.72 \\
\hline Beta-blocker use & $1(17 \%)$ & $1(10 \%)$ & 0.78 \\
\hline Calcium-channel blocker use & $0(0 \%)$ & $2(20 \%)$ & 0.24 \\
\hline Lipid-lowering therapy use & $1(17 \%)$ & $2(20 \%)$ & 0.88 \\
\hline
\end{tabular}

a Mean values \pm SD are given for continuous variables, and number of patients (with percentages in parentheses) is given for discrete variables.

ther found that cardiac medication use (which was infrequent in our population) did not significantly predict MBF values. We also found no significant association between self-reported moderate physical activity and endothelium-dependent MBF in response to CPT $(0.91 \pm 0.23$ vs. $1.12 \pm 0.33, p=0.20)$. However, women who reported regular, at least moderate physical activity had a significantly higher percentage increase in maximal, adenosine-induced MBF and, consequently, a significantly higher MFR than those who did not report regular exercise (Fig. 1). This was due to higher resting $\mathrm{MBF}$ in the inactive group as well as lower adenosine-induced MBF in these women. There was no significant difference in weight between those who exercised regularly and those who did not (172 \pm 28 vs. $179 \pm$ $28 \mathrm{lbs}, p=0.6)$.

Table 2. Myocardial Blood Flow Results for Each Patient

\begin{tabular}{|c|c|c|c|c|c|c|c|}
\hline Subject & $\begin{array}{c}\text { Resting } \\
\operatorname{RPP}^{a}\end{array}$ & $\begin{array}{c}\text { Resting } \\
M B F\end{array}$ & $\begin{array}{l}C P T \\
R P P\end{array}$ & $\begin{array}{l}C P T \\
M B F\end{array}$ & $\begin{array}{c}\text { Adenosine } \\
\text { RPP }\end{array}$ & $\begin{array}{c}\text { Adenosine } \\
\text { MBF }\end{array}$ & $M F R$ \\
\hline \multicolumn{8}{|c|}{ Active subjects } \\
\hline 1 & 6,985 & 0.8325 & 7,686 & 1.334 & 10,622 & 2.890 & 3.472 \\
\hline 2 & 8,576 & 0.8444 & 9,768 & 0.850 & 13,328 & 2.800 & 3.316 \\
\hline 3 & 10,950 & 1.3024 & 11,696 & 0.878 & 18,408 & 3.093 & 2.375 \\
\hline 4 & 7,296 & 0.6261 & 11,468 & 0.878 & 12,371 & 3.644 & 5.819 \\
\hline 5 & 9,956 & 1.0067 & 11,932 & 0.895 & 16,906 & 3.265 & 3.243 \\
\hline 6 & 8,241 & 0.7188 & 9,313 & 0.632 & 14,280 & 2.771 & 3.856 \\
\hline \multicolumn{8}{|c|}{ Inactive subjects } \\
\hline 1 & 10,360 & 1.0693 & 12,236 & 1.356 & 12,998 & 1.175 & 1.099 \\
\hline 2 & 6,534 & 0.7926 & 8,136 & 0.705 & 8,648 & 2.080 & 2.624 \\
\hline 3 & 7,800 & 1.1375 & 9,660 & 1.448 & 12,555 & 2.660 & 2.339 \\
\hline 4 & 9,734 & 1.3674 & 12,410 & 1.324 & 13,528 & 1.520 & 1.112 \\
\hline 5 & 10,413 & 0.9084 & 9,940 & 0.958 & 13,440 & 4.563 & 5.023 \\
\hline 6 & 14,469 & 1.2250 & 16,650 & 1.119 & 16,789 & 1.314 & 1.073 \\
\hline 7 & 15,015 & 1.4545 & 14,832 & 1.146 & 18,624 & 2.693 & 1.851 \\
\hline 8 & 8,658 & 1.1906 & 11,703 & 1.444 & 14,522 & 3.563 & 2.993 \\
\hline 9 & 10,360 & 1.1976 & 12,324 & 1.203 & 16,695 & 3.455 & 2.885 \\
\hline 10 & 8,856 & 0.8999 & 8,436 & 0.449 & 12,103 & 1.711 & 1.901 \\
\hline
\end{tabular}

aRPP, rate pressure product (maximal heart rate $\times$ maximal systolic blood pressure); MBF, myocardial blood flow in $\mathrm{mL} / \mathrm{kg} / \mathrm{min}$; CPT, cold pressor test; MFR, myocardial flow reserve. 
Table 3. Mean Myocardial Blood Flow Results ${ }^{a}$

\begin{tabular}{lccc}
\hline & $\begin{array}{c}\text { Active group } \\
n=6\end{array}$ & $\begin{array}{c}\text { Inactive group } \\
n=10\end{array}$ & p value \\
\hline Resting rate pressure product & $8,667 \pm 1,534$ & $10,220 \pm 2,690$ & 0.22 \\
Resting MBFb & $0.89 \pm 0.24$ & $1.12 \pm 0.21$ & 0.06 \\
CPT rate pressure product & $10,311 \pm 1,677$ & $11,633 \pm 2,709$ & 0.30 \\
CPT MBF & $0.91 \pm 0.23$ & $1.12 \pm 0.33$ & 0.20 \\
Adenosine rate pressure product & $14,319 \pm 2,893$ & $13,990 \pm 2,840$ & 0.83 \\
Adenosine MBF & $3.08 \pm 0.34$ & $2.47 \pm 1.12$ & 0.22 \\
MFR & $3.68 \pm 1.16$ & $2.29 \pm 1.20$ & 0.04 \\
\hline
\end{tabular}

aValues are given as mean $\pm \mathrm{SD}$.

${ }^{b} \mathrm{MBF}$, myocardial blood flow in $\mathrm{mL} / \mathrm{kg} / \mathrm{min}$; CPT, cold pressor test; MFR, myocardial flow reserve.

\section{DISCUSSION}

In the present study, self-reported, moderate or greater regular physical activity was associated with a significantly higher percentage increase from rest in adenosine-induced MBF and, consequently, a significantly higher MFR, when compared with results in sedentary women. Adenosine effects on the myocardium can be characterized as a composite index of vascular reactivity. CHD risk

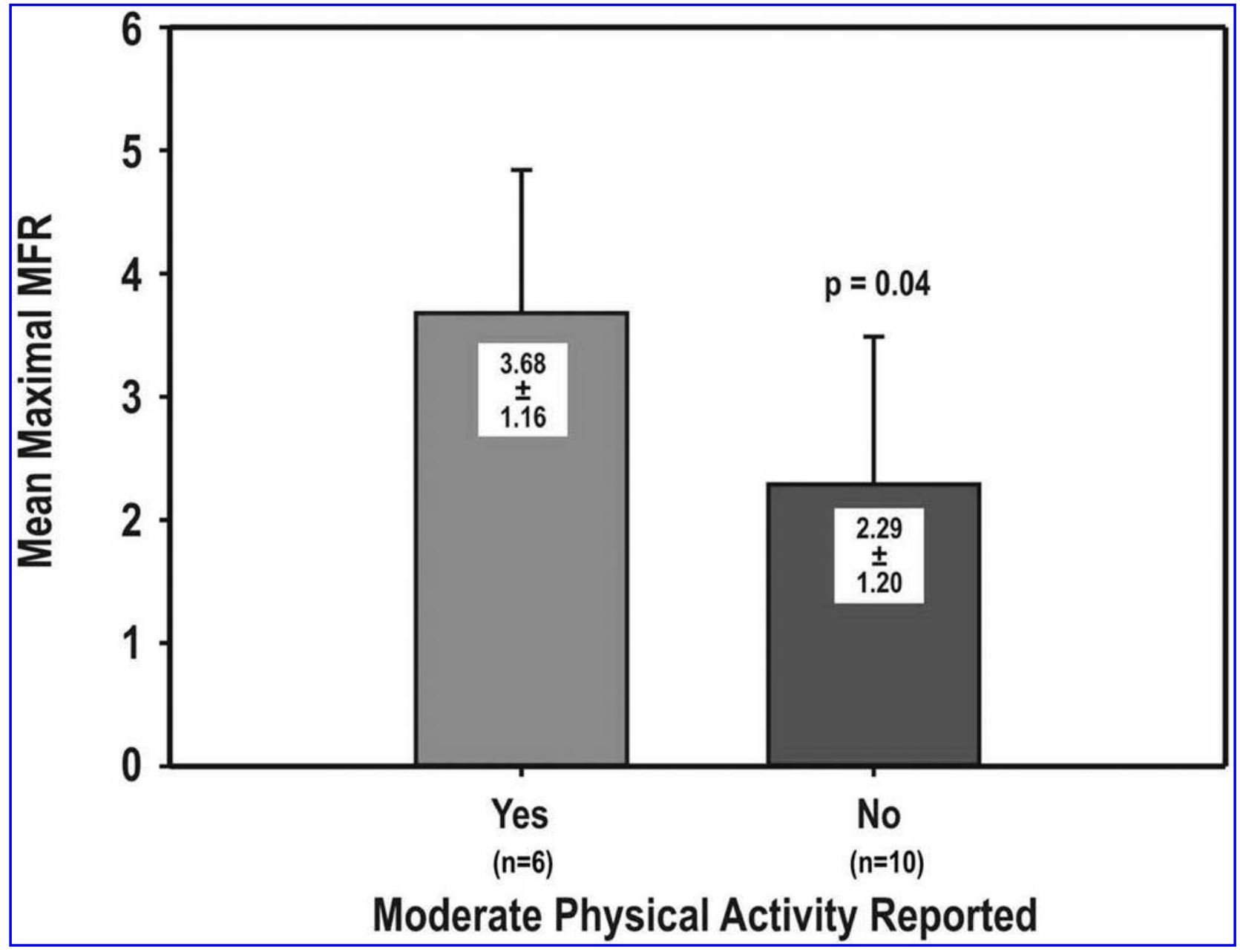

FIG. 1. Women who reported regular, at least moderate physical activity had a significantly higher mean maximal myocardial flow reserve (MFR) than those who reported no regular exercise (3.68 \pm 1.16 vs. $2.29 \pm 1.20, p=0.04)$. 
factors, such as hypercholesterolemia, hypertension, smoking, family history of premature CHD, and obesity, have all been associated with impaired vascular reactivity, indicating abnormal endothelial function. ${ }^{7-12}$

Exercise has been shown to improve endothelium-dependent vasodilation in large resistance blood vessels. ${ }^{2}$ In one study of PET-derived MBF and MFR in individuals with cardiovascular disease (CVD) who participated in an intensive program of diet and exercise, resting cardiovascular workload and MBF decreased, whereas maximal blood flow increased, leading to significant improvements in MFR. ${ }^{13}$ Furthermore, individuals who are moderately physically fit have lower CVD mortality rates when compared with low fitness level individuals, no matter what other combination of CHD risk factors they may have. ${ }^{1}$ One analysis that stratified both men and women into low, moderate, and high levels of fitness on the basis of maximal treadmill exercise testing detected death rates per 10,000 woman-years of 40, 16 , and 7 for women in each fitness level, respectively. ${ }^{14}$ The American Heart Association has labeled physical inactivity a major risk factor for CVD, and has endorsed moderate physical exercise as one part of a lifestyle strategy designed to lower cardiovascular risk. ${ }^{15}$ Our study lends further support to this recommendation by providing a potential mechanism of benefit, namely, improved MFR. MFR has been shown by others to be a strong, independent predictor of cardiovascular events and mortality in several patient groups. Schächinger et al. ${ }^{16}$ invasively measured coronary flow reserve in response to acetylcholine in cardiac catheterization patients and found that impaired responses predicted increased CHD events. Cecchi et al. ${ }^{17}$ studied patients with hypertrophic cardiomyopathy using PET in combination with dipyridamole infusion and found that impaired MFR predicted adverse cardiovascular outcomes and death. ${ }^{17}$

\section{Study limitations}

Our results are limited by the cross-sectional design, which does not allow us to assume a causal relationship between physical activity and MBF. Our questions concerning physical activity were qualitative and imprecise and relied on subjects' recall. However, the questionnaire we used has been validated and is widely used. Our study population consisted mainly of a relatively inac- tive, overweight and obese population with multiple risk factors for CHD. Certainly, individuals who do not exercise are more likely to be overweight or obese, so it is almost impossible to completely separate the impact of overweight and obesity from physical activity. We did not include a sufficient number of age-matched athletes or subjects who did not have CHD risk factors. Another limitation is our small sample size, which did not allow us to adequately correct for multiple variables or to show significant correlations between MBF and traditional CHD risk factors.

\section{CONCLUSIONS}

Our findings suggest that moderate physical activity is associated with greater MFR in healthy postmenopausal women with risk factors for CHD because of decreased resting cardiovascular workload and blood flow and increased maximal MBF. As a result, moderate physical activity may improve prognosis in these women with respect to long-term cardiovascular events and mortality. Our study provides further mechanistic support for the beneficial cardiovascular effects of exercise.

\section{ACKNOWLEDGMENTS}

We thank the technologists of the PET Suite at the University of Michigan: Paul Kison, Ed McKenna, Jill Rothley, and Andrew Weeden. We also thank Daniel Montgomery for his assistance with statistical analysis and graphics.

\section{REFERENCES}

1. Blair SN, Kampert JB, Kohl HW 3rd, et al. Influences of cardiorespiratory fitness and other precursors on cardiovascular disease and all-cause mortality in men and women. JAMA 1996;276:205.

2. Gaenzer H, Neumayr G, Marschang P, Sturm W, Kirchmair R, Patsch JR. Flow-mediated vasodilation of the femoral and brachial artery induced by exercise in healthy nonsmoking and smoking men. J Am Coll Cardiol 2001;38(5):1313.

3. Paffenbarger RS Jr, Wing AL, Hyde RT. Physical activity as an index of heart attack risk in college alumni. Am J Epidemiol 1978;108:161.

4. McKechnie R, Rubenfire M, Mosca L. Association between self-reported physical activity and vascular re- 
activity in postmenopausal women. Atherosclerosis 2001;159:483.

5. Duvernoy C, Martin J, Briesmiester K, Bargardi A, Muzik O, Mosca L. Myocardial blood flow and flow reserve in response to hormone therapy in postmenopausal women with risk factors for coronary disease. J Clin Endocrinol Metab 2004;89:2783.

6. Martin JW, Briesmiester K, Bargardi A, Muzik O, Mosca L, Duvernoy CS. Weight changes and obesity predict impaired resting and endothelium-dependent myocardial blood flow in postmenopausal women. Clin Cardiol 2005;28:13.

7. Dayanikli F, Grambow D, Muzik O, Mosca L, Rubenfire M, Schwaiger M. Early detection of abnormal coronary flow reserve in asymptomatic men at high risk for coronary artery disease using positron emission tomography. Circulation 1994;90:808.

8. Frielingsdorf J, Kaufmann P, Seiler C, Vassalli G, Suter T, Hess OM. Abnormal coronary vasomotion in hypertension: Role of coronary artery disease. I Am Coll Cardiol 1996;28:935.

9. Celermajer DS, Sorensen KE, Georgakopoulos D, et al. Cigarette smoking is associated with dose-related and potentially reversible impairment of endothelium-dependent dilation in healthy young adults. Circulation 1993;88:2149.

10. Nitenberg A, Valensi P, Sachs R, Dali M, Aptecar E, Attali JR. Impairment of coronary vascular reserve and ACh-induced coronary vasodilation in diabetic patients with angiographically normal coronary arteries and normal left ventricular systolic function. Diabetes 1993;42:1017.

11. Al Suwaidi J, Higano ST, Holmes DR Jr, Lennon R, Lerman A. Obesity is independently associated with coronary endothelial dysfunction in patients with normal or mildly diseased coronary arteries. I Am Coll Cardiol 2001;37:1523.
12. Seiler C, Hess OM, Buechi M, Suter TM, Krayenbuehl HP. Influence of serum cholesterol and other coronary risk factors on vasomotion of angiographically normal coronary arteries. Circulation 1993;88:2139.

13. Czernin J, Barnard RJ, Sun KT, et al. Effect of shortterm cardiovascular conditioning and low-fat diet on myocardial blood flow and flow reserve. Circulation 1995;92:197.

14. Blair SN. Physical inactivity and cardiovascular disease risk in women. Med Sci Sports Exerc 1996;28:9.

15. Thompson PD, Buchner D, Pina IL, et al. Exercise and physical activity in the prevention and treatment of atherosclerotic cardiovascular disease: A statement from the Council on Clinical Cardiology (Subcommittee on Exercise, Rehabilitation, and Prevention) and the Council on Nutrition, Physical Activity, and Metabolism (Subcommittee on Physical Activity). Circulation 2003;107:3109.

16. Schächinger V, Britten MB, Zeiher AM. Prognostic impact of coronary vasodilator dysfunction on adverse long-term outcome of coronary heart disease. Circulation 2000;101:1899.

17. Cecchi F, Olivotto I, Gistri R, Lorenzoni R, Chiriatti G, Camici PG. Coronary microvascular dysfunction and prognosis in hypertrophic cardiomyopathy. $\underline{\mathrm{N}}$ Engl J Med 2003;349:1027.

Address reprint requests: Claire S. Duvernoy, M.D.

Veterans Affairs Ann Arbor Healthcare System University of Michigan Health System 2215 Fuller Road, IIIA Ann Arbor, MI 48105

E-mail: duvernoy@umich.edu 